\title{
Conflicto por el agua en el Sistema de Riego Tepetitlán después de la trasferencia
}

\author{
Acela Montes de Oca Hernández* \\ Cristina Chávez Mejía ${ }^{* *}$ \\ Francisco Guízar Vázquez ${ }^{* *}$ \\ Ivonne Vizcarra Bordi ${ }^{* *}$
}

Resumen: El objetivo del artículo es identificar y explicar el conflicto surgido en el Sistema de Riego Tepetitlán, Estado de México, a partir de la trasferencia de los distritos de riego a los usuarios, en 1993, consistente en la entrega de obras de infraestructura. El Sistema de Riego Tepetitlán fue trasferido según la modalidad de unidad de riego, poco conocida en los estudios en México. Aquí se analiza este sistema bajo la lente del conflicto; se identifica y examina los momentos coyunturales que lo propician en las organizaciones de regantes, y se explica la situación actual de administración, operación y manejo del regadío.

Palabras clave: conflicto, distrito de riego, unidad de riego, sistema de riego, organizaciones de regantes, administración.

\footnotetext{
* Doctora en ciencias agropecuarias y rurales, Universidad Autónoma del Estado de México. Carretera Toluca-Ixtlahuaca, km 15. Teléfono (722) 295 5552. Correo electrónico:

amhplata2007@yahoo.com.mx

** Profesores-investigadores del Instituto de Ciencias Agropecuarias y Rurales, Universidad Autónoma del Estado de México. Correos electrónicos: cchavezm@uaemex.mx / fguizarv@, gmail.com / ivbordi@yahoo.com.mx
} 
Abstract: This article identifies and explains the conflict which emerged in the Tepetitlán irrigation system in the state of Mexico when the government transferred control of the irrigation districts to the consumers. The Tepetitlán irrigation system was part of Irrigation District 033 and managed by the government until 1993, at which point the government transferred management and infrastructure maintenance to the local community. The transfer from Irrigation District to Irrigation Unit-a topic which has hardly been studied in Mexico-is analyzed using conflict theory. We analyze the situations that cause conflict in local organizations. Based on this, we explain the present management situation of the irrigated land.

Keys words: conflict, irrigation district, irrigation unit, irrigation system, irrigator organizations, management.

\section{Introducción}

La preocupación por el agua tiene varias aristas, entre ellas la más importante en los últimos años ha sido la escasez. Numerosas instituciones internacionales promulgan y defienden una mejor y justa gestión del recurso hídrico, cuyos usos son el industrial, doméstico y agrícola. El agua utilizada en la agricultura genera sistemas de valores propios en cada pueblo. En dichos sistemas se incluyen los conocimientos y prácticas de los actores sociales que interactúan en el aprovechamiento del agua en la agricultura, sector en el que cada uno de ellos cumple un papel importante como gestor o administrador. La gestión, sin embargo, como instrumento político, queda supeditada a las políticas agrícolas del mundo y del país, tal es el caso de los denominados distritos de riego, que en México fueron creados por el Estado a partir de 1926; algunos se entregaron a los usuarios durante la década de 1940, y el resto fue administrado por personal burócrata (contratado por el Estado), hasta 1992. El objetivo de los distritos de riego fue aprovechar el agua con la cons- 
trucción de obras hidráulicas para almacenar el recurso, y después encauzarlo y hacerlo llegar a los lugares que necesitaban del riego. Dicha tarea estuvo a cargo de la Comisión Nacional de Irrigación, en 1926, y fue decisiva para el impulso económico y social del país.

La creación de los distritos mejoró el campo agrícola, después de la Revolución Mexicana ayudó a resolver el problema agrario en algunas zonas del país, sobre todo en el norte (Rendón Pimentel 2008). Los distritos de riego fueron administrados, manejados y operados por el gobierno federal, razón por la cual también se les denominó gran regadío. Los estudios sobre sistemas de riego hasta el año 2000 se enfocaron a la gran irrigación, construida y administrada por el Estado (Palerm et al. 2000, 32).

Los distritos de riego en México no son homogéneos, presentan peculiaridades. Palerm $(2008,55)$ realiza un estudio interesante sobre sus características, y menciona que no todos fueron construidos por el Estado, el cual los tomó o los amplió. Algunas obras que ya existían fueron consideradas como distritos de riego en 1926, en el momento en que el Estado las administra, como el caso del 033, al que perteneció la presa de Tepetitlán (edificada con capital privado desde la época de la Colonia); otros tenían una organización autogestiva (unidades de riego), antes de que el Estado tuviera injerencia administrativa.

Dichas unidades son sistemas de riego ${ }^{1}$ pequeños y medianos que han sido gobernados y administrados por los propios regantes desde el reparto agrario (1934-1940), antes por haciendas y marginalmente por comunidades campesinas. En México, las unidades de riego concentran tres millones de hectáreas de riego, carecen de personal especializado; son los propios regantes quienes ocupan los puestos necesarios, con cargos honoríficos y en ocasiones pagados, como jueces de aguas, atopile, comisionado del agua, regidor del

\footnotetext{
${ }^{1}$ Para Hunt (1997), un sistema de riego es el curso del agua a partir de una toma hasta la parcela. En el regadío se pueden encontrar varios sistemas de riego, que denotan formas de organización distintas. Para Kelly $(1983,81)$ es "un arreglo por el cual el agua se lleva de una fuente a un área que necesita agua para facilitar la producción de los cultivos deseados”. Por su parte, Cressier (1995) prefiere hablar de red hidráulica, en ella engloba no sólo a la parte tecnológica y agrícola, también a la organización social necesaria para irrigar los campos de cultivo.
} 
agua, presero, rejero y otras designaciones locales para llevar a cabo las tareas que impone el sistema de riego (Martínez y Palerm 1997, 13; Palerm et al. 2000; Palerm 2000a; Palerm 2005b, 5).

En este tenor, la pregunta que guía el artículo es ¿por qué en el Sistema de Riego Tepetitlán se practica una administración distinta a la marcada por el Estado después de la trasferencia, y de qué manera ello origina conflicto en el regadío?

La trasferencia de los distritos de riego a los usuarios consistió en la entrega de la red menor del sistema de riego (canales de riego, caminos, drenes, compuertas) a una asociación civil, mientras que la red mayor (presa y derivadora) quedó en manos del Estado. Una vez aceptada la trasferencia, la asociación civil asumía la administración, operación y manejo de las obras concesionadas (Pérez 1994). Existieron dos prototipos de trasferencia, en la primera la asociación contrataría al personal gerencial para la administración, operación y manejo de la infraestructura y en la segunda, los usuarios asumirían los cargos de dicho sistema.

Con la trasferencia, la propuesta del gobierno federal para el manejo del agua fue un cambio de administración, hasta ese momento a cargo del Estado, en la cual los usuarios manejarían parte del sistema. La Comisión Nacional del Agua (CONAGUA), siguiendo los lineamientos de la Ley de Aguas, continuó con la administración gerencial que antes poseían los sistemas de riego. La diferencia, después de 1992, radicó en que los usuarios formados en asociaciones civiles serían quienes contratarían y le pagarían al personal técnico (gerentes, canaleros, secretarias). Palerm (2005b) analizó este proceso de trasferencia, y encontró un cambio en la autoridad, pero no en el personal a cargo de las obras, en algunos distritos de riego que seguían contando con apoyo técnico y financiero de la federación. En otros, como es el caso de estudio, no se aceptó este tipo de "burocracia hidráulica”, como la denomina Wittfogel (1966), y fueron etiquetados por el Estado como unidades de riego.

Para los usuarios de Tepetitlán, pertenecer a unidades de riego implicó aprender a administrar el sistema sin apoyo técnico y financiero de la conAGUA. En cuanto al manejo del sistema, los usuarios tenían conocimiento previo, pues al parecer cuando el Estado crea 
el distrito de riego 033 (al que fue incluido el Sistema de Riego Tepetitlán) en 1946, ellos se organizaban para realizar las tareas de limpieza y mantenimiento de la red de canales principales y secundarios. Las unidades de riego, como la de Tepetitlán, carecen de personal técnico y de apoyo financiero del Estado y son administradas, operadas y manejadas por los usuarios. Ésta se integra por los municipios de San Felipe del Progreso, Ixtlahuaca y Jocotitlán, del Estado de México, cuyos usuarios son campesinos mazahuas bilingües; el Sistema de Riego Tepetitlán se localiza en la cuenca alta LermaSantiago; se conforma por 31 ejidos y 15 pequeñas propiedades, e irriga un promedio de 9750 hectáreas, en las que se cultiva, en orden de importancia, maíz, tomate, cebada, haba, calabaza y frijol; el agua es utilizada como punta de riego y como riego auxiliar.

Los usuarios de Tepetitlán no aceptaron seguir el modelo tecnocrático que dictó el Estado en 1992 (Wittfogel 1966; Palerm 2000b; Palerm 2005a), pues el modelo de trasferencia implementado indicaba un aumento a la cuota de riego, de 10 a 90 pesos (1 a 8 dólares). Pasar de distrito a unidad de riego originó problemas entre usuarios y la CONAGUA, que se reflejaron en las comunidades beneficiadas con el sistema. A partir de lo anterior, el conflicto se aborda desde una visión coyuntural marcada por la política de trasferencia.

El conflicto social es parte inherente a toda sociedad, cuya perspectiva, desde el punto de vista de Rosseau, es armónica y su integración es resultado del consenso de sus elementos. La visión de Hobbes dista de esta aseveración al marcar la idea de discordia social, y la integración sólo es posible por la vía de la coacción (Giner 2009).

En sistemas de regadío, el conflicto es inherente a las relaciones sociales que se tejen para la administración del recurso. Maass y Anderson $(1997,265)$ en siete estudios de caso en España y Estados Unidos destacan que la organización para el regadío es necesariamente muy cohesiva y el individuo tiene que someterse a la colectividad, que el regadío es fuente inevitable de conflicto y la organización sustentable es capaz de contenerlo, los regantes cuentan con procedimientos e instituciones operativos que desalientan los conflictos y resuelven los que surjan. 
Otras posturas teóricas del conflicto tratan de investigar su naturaleza y estructura buscando afanosamente los factores y actores que promueven inestabilidad en los sistemas sociales, así como los procedimientos para afrontarlos (Gelik 1993 y 1994; Wolf et al. 2005, 157). De esta manera, el conflicto en el riego puede dar la idea de un desmantelamiento de la infraestructura hidráulica adjudicada, claro está, a determinados actores, de modo que significa un riesgo que impide la continuidad del regadío.

Sin el objeto de caer en los extremos al tratar al Sistema de Riego en Tepetitlán como uno armonioso o destructivo, es preferible ahondar en las propuestas alternativas, como la teoría del conflicto (Millon et al. 1997), que si bien acepta que en todo sistema de riego existe el conflicto, también reconoce que hay cohesión y cooperación. Para Fernea (1997), la cohesión en el regadío está ligada a la responsabilidad que asumen los regantes para decidir sobre la administración del sistema y controlarlo, ello requiere la participación de los actores (los regantes, las autoridades locales, las de la asociación civil y el Estado) en la toma de decisiones.

Para que exista cooperación en el regadío es necesario que los usuarios tengan intereses comunes, el recurso que los une en el caso estudiado es el agua y el común denominador que los liga es el acceso a ella, su distribución y el mantenimiento del sistema. Pero los intereses de cada regante son divergentes, por ello la cohesión es necesaria en el regadío para mantener el control en el sistema, y porque facilita los acuerdos.

Lo interesante del estudio del conflicto, con su contraparte que es la cohesión y la cooperación, es que permite describir y analizar las instancias de los regantes, para enfrentar las dificultades en el regadío, después del retiro del Estado. Por ello, es útil destacar la estructura del regadío, desde el nivel macro (Estado) hasta el local (organizaciones de regantes), para entender el proceso organizativo cohesivo y cooperativo para detener o minimizar el conflicto.

La organización para el aprovechamiento del recurso agua en el sistema Tepetitlán requiere la cooperación de los usuarios para administrarlo, que incluye la rehabilitación, mantenimiento y limpia de la infraestructura, control de problemas y la distribución del agua, que en parte se hace siguiendo el esquema marcado por el 
Estado antes de la trasferencia, pero dicho esquema de distribución es ajustado a las necesidades de los propios regantes, que son básicamente tener acceso al agua para el riego oportuno antes de la siembra y el buen desarrollo de los cultivos. El funcionamiento del riego exige una organización social cohesiva y cooperativa (Martínez y Palerm 1997).

Para analizar el conflicto, el enfoque se pondrá en la estructura organizativa del Sistema de Riego Tepetitlán y su funcionamiento después de su trasferencia. En primer lugar, el Estado cataloga como conflictivo al sistema Tepetitlán, aludiendo ingobernabilidad por parte de los regantes. En segundo, la asociación civil atribuye el conflicto a la presencia de personal burócrata. La situación es distinta en los ejidos y pequeñas propiedades, donde hay una fortaleza organizativa que detiene el conflicto. En este sentido, el sistema Tepetitlán constituye la respuesta de los regantes a la situación problemática entre Estado y asociación civil, entre ésta y las autoridades locales del riego y de éstas con los usuarios por la administración del regadío.

\section{Metodología}

La investigación en campo comprendió dos etapas; en la primera se realizó un sondeo, durante los fines de semana de febrero a mayo de 2008, para identificar el espacio de riego y autoridades a cargo de la administración del sistema. La segunda se dedicó al trabajo etnográfico en los tres municipios que conforman el sistema, de julio de 2008 a mayo de 2009; se observó el manejo del sistema y la operación, desde el ejido al consejo directivo.

De octubre de 2008 a mayo de 2009 se hicieron recorridos para observar la administración del sistema, que conlleva una serie de acciones del consejo directivo de la asociación civil ante la gerencia regional y estatal de la conAGUA, como la solicitud de volumen de agua al inicio del riego y petición de incremento o decremento de volumen en época alta, media y final del regadío. Mediante un recuento histórico se establecieron conexiones entre el antes y el 
ahora, lo que permitió esclarecer los conflictos, en específico en la administración. También se realizaron entrevistas a profundidad con autoridades de la jefatura de distrito de riego 033, en el Estado de México, y comunitarias (dos consejos directivos presentes como asociación civil), para entender la administración, operación y manejo en el sistema.

\section{El Sistema de Riego Tepetitlán}

El Sistema de Riego Tepetitlán constituye un espacio hidráulico del cual existen antecedentes documentales de que formó parte del distrito de riego 033 en 1946, aunque no existe el decreto de creación (Montes de Oca et al. 2010). Dicho distrito comprende los municipios Temascalcingo, Acambay, Atlacomulco, San Felipe del Progreso, Ixtlahuaca y Jocotitlán, del Estado de México, otro de Querétaro y uno más de Michoacán. Como distrito de riego 033, el Sistema de Riego Tepetitlán fue integrado a los municipios mencionados, los cuales no mantenían relación física alguna como se muestra en la figura 1.

La presa Tepetitlán se encuentra dentro de la cuenca hidrológica Lerma-Chapala-Santiago, su extensión de 132724 km² representa cerca de 7 por ciento del territorio nacional (CONAGUA 2002). La subcuenca de la presa tiene una superficie de $368.74 \mathrm{~km}^{2}, 0.69$ por ciento del total de la cuenca Lerma-Chapala y posee un área de captación de $207.63 \mathrm{~km}^{2}$, estos datos la ubican como la cuarta presa más importante del Estado de México (Wester et al. 2004). Esta infraestructura hidráulica implica el funcionamiento de la presa y su derivadora en manos de personal calificado por la CONAGUA, que se auxilia de un presero y dos aforadores, empleados federales que controlan el volumen de agua que llega a la presa, al río y a los canales (entrevista al ingeniero Luis Eduardo Mejía Pedrero, subgerente de Ingeniería, gerencia estatal, en el Estado de México, 6 de marzo de 2009). El Sistema de Riego Tepetitlán (módulo II) cuenta con el mayor número de hectáreas regadas y de usuarios beneficiados, comparado con los otros módulos de riego, que integran al distrito 033 (véase figura 2). 


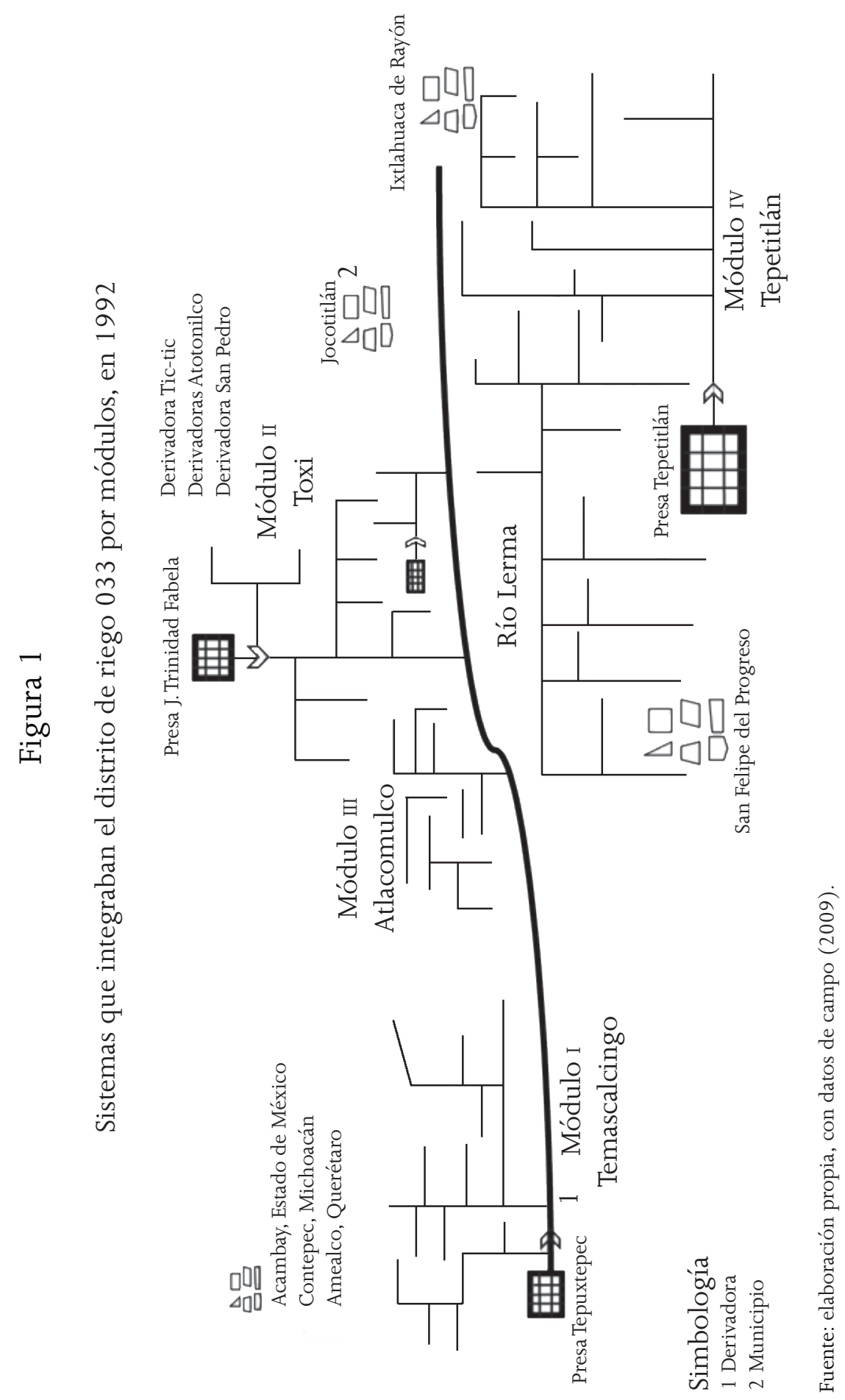




\section{Figura 2}

Módulos de riego del distrito 033, Estado de México

\begin{tabular}{|l|l|c|c|}
\hline Módulo & \multicolumn{1}{|c|}{ Municipio } & $\begin{array}{c}\text { Número de } \\
\text { hectáreas }\end{array}$ & $\begin{array}{c}\text { Número de } \\
\text { usuarios }\end{array}$ \\
\hline I & Temascalcingo & 4601 & 4023 \\
\hline II & Tepetitlán & 9720 & 8568 \\
\hline III & Toxi & 637 & 645 \\
\hline IV & Atlacomulco & 2732 & 3735 \\
\hline Total & & 17738 & 14555 \\
\hline
\end{tabular}

Fuente: Archivo de la Jefatura de Distritos de Riego, "Resumen de la organización para la operación del distrito de riego 033”, 1999. Estado de México.

\section{La política legislativa de trasferencia de distritos de riego y la organización de regantes en Tepetitlán}

La política de trasferencia de distritos de riego significó un reordenamiento administrativo. Para trasferir el distrito de riego 033 a los usuarios, se dividió en cuatro módulos; I (Temascalcingo), III (Toxi) y IV (Atlacomulco) aceptaron la trasferencia según los lineamientos de la CONAGUA, pero el módulo iI (Tepetitlán) rehusó la propuesta del Estado de incrementar la cuota de riego y designar a una asociación civil para continuar con el tipo de personal gerencial.

En el módulo II, los regantes se niegan a seguir este esquema de trasferencia desde 1993, a pesar de ello la CONAGUA integra una asociación civil, bajo un modelo gerencial, y contrata a una empresa para que informe a los usuarios (mediante ciclos de conferencias y entrega de folletos), sobre la diferencia entre seguir siendo distritos de riego y dejar de serlo (Murillo y Romero 2000). La información no fue precisa, las dudas siempre quedaron latentes, y a pesar de ello un grupo de usuarios pendientes de estas pláticas y conferencias decide solicitarle a la conAGUA les trasfiera el módulo in Tepetitlán bajo otra modalidad.

La CONAGUA establece el término unidad de riego, ante la negativa de los usuarios de pagar al personal gerencial, y eso tuvo significados distintos entre los implicados en la trasferencia; la CONAGUA 
lo veía como apoyos económicos gubernamentales derogados y carencia de personal técnico para operar el sistema (entrevista al ingeniero Raúl Rivas Castillo, jefe de Distritos de Riego del Estado de México); para la asociación civil, que los usuarios se encargarían del manejo, administración y operación con apoyo técnico de la coNAGUA; para los usuarios, que el agua de la presa Tepetitlán era suya y deberían conservarla, sobre todo representaba que el agua tenía dueño ${ }^{2}$ (entrevista al señor Castro, presidente de la asociación civil Unión Rural de la Presa Tepetitlán, de 2002-2004).

Una vez aceptada la trasferencia como unidad de riego, la administración del sistema de riego implicaba la elección de otra asociación civil y la destitución de la primera, instituida por el Estado. La nueva asociación constituida por los usuarios tendría la obligación de contratar al personal especializado, pagado por el Estado hasta 1993, que comprendía al gerente, subjefe de operación, jefe de los canaleros y los mismos canaleros, y cubriría sus honorarios, que para hacerlo debería incrementar la cuota de cobro, tal y como lo indicaba la CONAGUA, situación que generó molestias en los usuarios, que se negaron a seguir este mandato gubernamental. De este modo, el conflicto presenta cuatro fases; la primera, el aumento de cuota de riego suscitó el problema en el sistema de riego trasferido por el Estado a los usuarios; en la segunda, el malestar se incrementa por la negativa del Estado a la petición de los regantes de bajar la cuota de riego de 90 a 20 pesos. Pero además, el Estado había integrado una asociación civil para trasferir el módulo in del distrito de riego 033 sin consultar a las autoridades tradicionales (comisariados ejidales), con el subsecuente aumento de cuota de riego. En la tercera fase, los usuarios piden al Estado destituir a la asociación civil que habían formalizado y que acepte una nueva conformada por los regantes, sin intervención del personal burocrático. En esta etapa se promovió el inicio de las negociaciones entre regantes y Estado; éstos exigieron a las autoridades retirarse del espacio de riego

2 El señor Zaragoza, del Grupo Mextepec, registró ante notario público a la asociación civil “Unidad de Riego Tepetitlán”, en 1999 y en el año 2000 recibió de la conAGUA el título de concesión. Una vez realizada la trasferencia, 70 por ciento de los usuarios lo aceptaron, debido a que el señor Zaragoza promovió el no aumento de la tarifa de riego impuesta por el Estado. 
durante las elecciones, para formalizar una nueva asociación civil y no prolongar más la trasferencia, situación que el personal burócrata aceptó pues debería entregar el sistema de riego de una u otra forma (entrevista al ingeniero Jorge Aurelio Monroy, subgerente de Operación de Infraestructura del distrito de riego 033).

La cuarta fase ocurrió dos años después de la trasferencia (2003), cuando un grupo de usuarios destituyó, con apoyo de autoridades del Estado, a la asociación civil que en 2001 recibió el título de concesión. El nuevo grupo de usuarios, también denominado asociación civil, recibió sólo la copia del título de concesión del Estado. De esta manera, el enfrentamiento entre las dos asociaciones civiles, surgido en 2003, se mantuvo hasta 2010.

A pesar del conflicto, las asociaciones civiles trataron de administrar el espacio de riego coordinándose con las autoridades de los ejidos y pequeñas propiedades que las legitimaban. Actualmente existen dos asociaciones civiles, una legitimada por usuarios y por el Estado y la otra sólo por usuarios, lo cual muestra formas de organización para la administración del riego. A pesar de ser un sistema de mediana irrigación, los usuarios tienen una organización social compleja a partir de la trasferencia, en 2001; son quienes ocupan los puestos que requiere la administración, operación y manejo del sistema de riego y red hidráulica, ${ }^{3}$ donde las comunidades cumplen una función determinada con ayuda de un consejo directivo y de las autoridades de ejidos y pequeñas propiedades. Pese a que el Sistema de Riego Tepetitlán, de ser distrito de riego (con apoyo financiero) pasó a unidad de riego (sin él), la asociación civil sigue trabajando a través de los enlaces entre organizaciones comunitarias y multicomunitarias, y tiene la obligación de administrar, manejar y operar el sistema de riego según sus propios estatutos.

La Unidad de Riego Tepetitlán, a nivel de ejidos y entre ejidos, presenta una forma de organización constantemente mejorada en el manejo del sistema de riego. Pero en cuanto a la operación del sistema administrado por las asociaciones civiles, conlleva severos conflictos que ocurren entre ellas porque cada una quiere legitimar

\footnotetext{
${ }^{3}$ Contrario a lo que dicta Vaidyanathan (2000), de la necesidad en estos sistemas multicomunitarios de personal especializado.
} 
su autoridad en espacios donde los usuarios no las reconocen, ello impacta directamente en la entrega de agua modificando las fechas o privilegiando a los usuarios que sí las legitiman. Sin embargo, este conflicto de inmediato lleva a las autoridades de los ejidos a organizarse de manera cohesiva y cooperativa, para minimizar el riesgo de quedar sin agua. Se forman grupos de 100 a 300 usuarios y no usuarios, ${ }^{4}$ y reclaman a las autoridades de la asociación civil regular el uso del agua, de manera que no favorezcan a unos ejidos sobre otros.

La organización de regantes a cargo del sistema es una asociación civil denominada “Unidad de Riego Tepetitlán”, está conformada por un consejo directivo y uno de vigilancia integrados por el presidente, secretario, tesorero y vocales. En la siguiente página se muestra el funcionamiento del organigrama.

El conflicto entre consejos directivos se agudiza cuando se enfrentan los que tienen a cargo cerca de 196 kilómetros de canales principales de riego y alrededor de 509 estructuras hidráulicas, y están en persistente jaloneo por la distribución del agua. Los problemas son llevados hasta el ámbito municipal, donde se solucionan mediante el pago de una multa o pena de cárcel a los infractores, sin que ello logre erradicarlos debido a que en cada ciclo de riego las pugnas sobre la distribución del agua están latentes. Es decir, cuando la estructura de autoridad de las asociaciones civiles busca en las autoridades municipales una minimización del conflicto, éstas no responden a sus expectativas, y las inducen a continuar con sus estrategias locales para detenerlo.

En la figura 3 se aprecia la participación de dos asociaciones civiles con sus respectivas autoridades, la señalada con línea continua está legitimada por el Estado y la indicada con línea punteada no está reconocida por él, pero sí por los usuarios. La participación de estos últimos como autoridades de las asociaciones civiles es importante, pues son los puentes de comunicación y negociación entre autoridades y usuarios para el reconocimiento del espacio de regadío.

\footnotetext{
${ }^{4}$ Los usuarios son los registrados en el padrón de la asociación civil y los no usuarios son sus familiares.
} 


\section{Figura 3}

Organigrama de la Unidad de Riego Tepetitlán A. C.

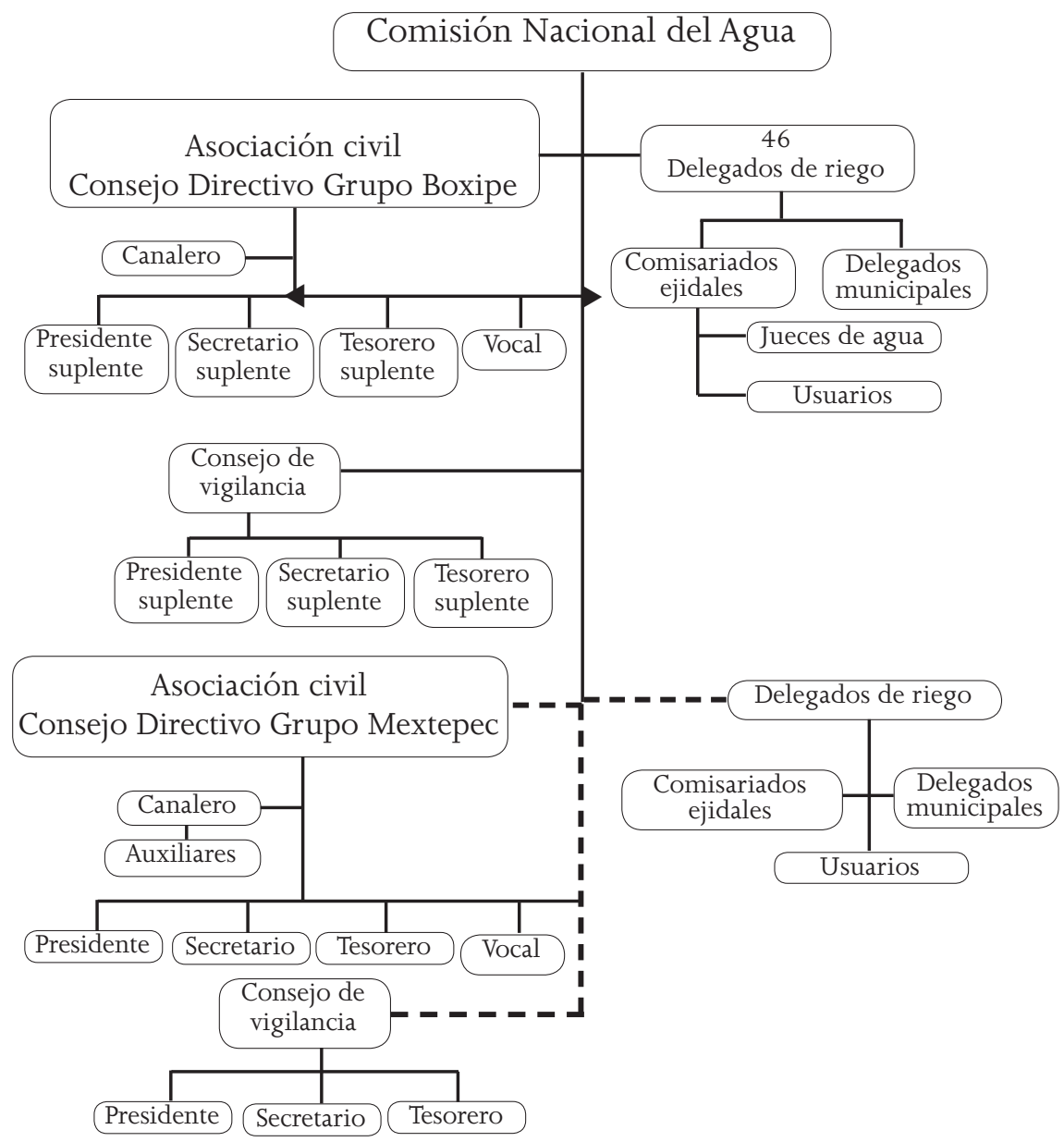

Fuente: elaboración propia, con datos de campo (2009). 
Las autoridades de la asociación civil se apoyan en las locales como los jueces de agua, delegados de riego, comisariados ejidales y delegados municipales para la administración del sistema de riego, lo que representa una descentralización de funciones y por ende una apropiación del sistema.

\section{La administración, operación y manejo de la red hidráulica de Tepetitlán: cohesión y cooperación}

La administración del sistema tiene dos tipos de gobierno, uno a cargo del Estado y el otro de los regantes; el personal de ambos cumple la tarea de planear, operar y controlar la infraestructura hidráulica, y asume la función de integrar y dirigir a los regantes para ejecutar el plan de riego, lo cual necesita una estructura jerárquica (Méndez 2000). De ser un sistema estatal, en 1993, -que funcionaba bajo un esquema jerárquico de una gerencia estatal, una jefatura de distritos de riego estatal con sus jefaturas de operación, administración, asistencia técnica y operativa pagada por el Estado- pasó a tener un diseño nuevo, donde los usuarios formaron una asociación civil para administrar el sistema de riego concesionado. "La primera Asociación Civil que sería la encargada de administrar el Sistema de Riego Tepetitlán, la formó la CNA en 1993 junto con otros funcionarios estatales" (entrevista al señor Garduño, delegado de riego de San Miguel Enyege). La intervención del Estado, al nombrar a los representantes de los usuarios ante él, tuvo repercusiones entre autoridades ejidales en la designación y en el proceso que implicó crear y formalizar una asociación civil.

La segunda asociación civil se formó en 1999 y pidió que se destituyera la primera, creada por el Estado; también le solicitó al personal gerencial que informara a cada ejido y pequeña propiedad sobre la trasferencia y el aumento de cuota de riego por parte del Estado, para que los usuarios tuviesen claro que no fue la asociación civil la que impuso la trasferencia del sistema ni la cuota de pago. Esa aclaración logró que 51 por ciento de los ejidos y pequeñas 
propiedades aceptaran a sus representantes. Por contar con más de 50 por ciento de aceptación de los usuarios, a la asociación civil se le trasfirió el Sistema de Riego Tepetitlán mediante un acta de concesión entregada por ConAGUA, que denominó unidad de riego “Unión Rural de la Presa Tepetitlán” A. C.

La tercera asociación civil, creada en 2001, pidió la destitución de la formalizada en 1999, argumentando que se autonombró. Y reclamaba que el nombre de "Unión Rural de la Presa Tepetitlán” A. C., se cambiara por el de "Grupo Tepetitlán", pero las autoridades de la Comisión Nacional del Agua se negaron, y les dijo a los usuarios que se quedaba tal y como se creó en 1999. El cambio aceptado por el Estado en 2002 fue destituir a las autoridades de los consejos directivo y el de vigilancia, a los que entregó el título de concesión, con lo que legitimó a la tercera asociación civil.

De 2001 a 2010 la administración del sistema de riego ha estado en manos de dos consejos directivos, que toman espacios de infraestructura hidráulica en las comunidades donde son legitimados. La existencia de dichos consejos en Tepetitlán se originó porque en 1999 hubo comunidades que no estuvieron de acuerdo con la elección del consejo directivo, y se organizaron y adoptaron el nombre de Grupo Mextepec.

Este cambio de diseño administrativo en el sistema originó la cooperación de sus integrantes en tres acciones: a) el enfrentamiento como usuarios ante el Estado, al no aceptar la trasferencia ni el incremento de cuota de riego; b) la formación de grupos sociales para la elección de sus representantes y c) la responsabilidad del sistema concesionado.

Esta negativa de los regantes a aceptar el incremento de la cuota provocó que quedaran fuera del distrito de riego 033. El cambio de distrito a unidad de riego causó siete años de antagonismos inter y extra comunitarios, originados por la formación de representantes de los 31 ejidos y 15 pequeñas propiedades ante la nueva figura institucionalizada, que se haría cargo del sistema de riego.

La CONAGUA propone que un solo comité directivo contrate a los ingenieros, técnicos y canaleros necesarios, para realizar todas las tareas de distribución. Sin embargo, este modelo contradice el diseño organizativo implementado por organizaciones campesinas, que 
Palerm (2005a) denomina como vernáculas, que parecen preferir la descentralización por niveles a tomar una posición de autoridades centralizadas.

La descentralización en el Sistema de Riego Tepetitlán obedece a los lazos de cooperación, desde el ejido hasta el consejo directivo de la asociación civil. Cada comunidad, a través de sus delegados de riego, realiza los trabajos de limpieza y conservación de los canales de riego, actividades que son acordadas en asambleas generales, que tienen la facultad de remover o ratificar total o parcialmente, en forma temporal o definitiva, a los miembros de los consejos directivo y de vigilancia por incumplimiento o violación a los estatutos, o, cuando así fuera considerado, para el logro del objeto social de la asociación.

En la convocatoria a asambleas comunitarias de ambos consejos, cuyo objetivo sea la destitución uno de otro, el Estado permanece como espectador y a la vez vigilante de las acciones que se presentan en el sistema, como lo refieren los delegados de riego. "En el momento en que se destituyó al Consejo Directivo de Mextepec, ingenieros de la CNA fueron llamados por el grupo de Boxipe para que pusieran orden en este asunto, y hasta vinieron policías municipales para que calmaran los ánimos entre estos dos grupos que quieren ser dueños de la presa" (entrevista al señor Sánchez, delegado de riego de San Isidro Boxipe).

Por su parte, las autoridades de la CONAGUA refieren:

Desconocemos cómo funciona ahora Tepetitlán, andan peleando dos grupos, no llegan a acuerdos, siempre están peleando y nosotros sólo nos enfocamos a darles el volumen de agua que solicitan y ya, no tienen un control del agua de ríos intermitentes que llegan a sus canales, desconocen el sistema, no tienen el conocimiento de la distribución, no realizan su plan de riegos (entrevista a Mejía Pedrero, subgerente de Ingeniería de la gerencia estatal de la Comisión Nacional del Agua).

La postura del Estado ante un módulo de riego desincorporado, como Tepetitlán, no concuerda con lo encontrado en el trabajo de 
campo, donde unos usuarios vigilan a otros durante la época de riego, pues todos necesitan del agua y deben hacer buen uso de ella, y no desperdiciarla. Cada usuario tiene el deber moral de informar cualquier anomalía que impida el aprovechamiento equitativo del agua, y si se violentan las reglas establecidas en las asambleas; por ejemplo, se debe reportar a cualquier persona de los ejidos que se observe rompiendo cadenas o abriendo compuertas (entrevista al señor Sabino). Esto porque la cooperación de usuarios y entre comunidades es necesaria para la intervención, participación y reciprocidad de los regantes en la administración, operación y manejo del sistema.

El consejo directivo, el comisariado ejidal, el delegado de riego y el juez de aguas son las autoridades que pueden imponer multas y castigos, por ejemplo dejar que los infractores rieguen al último. Otra de las funciones del consejo directivo, que requiere de la cooperación, es el manejo del sistema, incluida la limpieza y mantenimiento de canales y cobro de la cuota de pago por riego. "El responsable de organizar las fechas de limpieza y de entrega de canales limpios es el Consejo Directivo, en ellas deben cooperar todos los usuarios con ayuda de los comisariados ejidales y sus integrantes como el secretario, tesorero, vocales y suplentes" (entrevista al señor Nieves).

Por medio de perifoneo, los comisariados ejidales indican a los usuarios que la limpieza de canales inicia a finales de octubre y termina a mediados de enero, no todas las comunidades la realizan en una fecha determinada, pero para mediados de enero todos deben haber cumplido con esta tarea, porque en esa fecha y principios de febrero las autoridades comunitarias acuden al canal e inician el recorrido para "recibir los canales", que empieza en el límite entre ejidos. Después siguen el curso del canal que lleva el agua por gravedad hasta el final de los terrenos ejidales o de la pequeña propiedad. También los representantes de cada ejido designan los metros de canal por limpiar, y marcan los límites entre usuarios. El recorrido se realiza de 8:00 a 14:00 horas, y se van anotando tramo por tramo de canal a los usuarios que han hecho la limpieza.

Durante la entrega de canales, es común ver que algunos siguen trabajando, y hasta que terminan se les anota que han cumplido la 
tarea, los que ya la realizaron sólo deben acudir para verificar que las autoridades validen su esfuerzo.

\section{Cohesión y conflicto}

Existe la presencia de vínculos inter y extra comunitarios en la conservación de los canales en el Sistema de Riego Tepetitlán, que indudablemente están unidos por un recurso esencial que es el agua. En este sentido, el agua de la presa Tepetitlán que corre por los canales debe ser normada y dichas normas deben ser respetadas o tratar de que así sea.

El 12 de octubre de 2008 en la comunidad de San Pedro el Alto, el canal general margen derecha se derrumbó, un usuario dio aviso al juez de aguas, el juez de aguas me avisó y pedí el apoyo a los comisariados ejidales de los ejidos aledaños y rápidamente los usuarios de las comunidades de Santa Ana Ixtlahuaca, San Isidro Boxipe acudieron con palas y azadones para volver a colocar la pared de contención del canal y evitar que se siguiera quebrajando (entrevista al señor Bernardino, comisariado ejidal de Santa Ana La Ladera).

La distribución del agua depende del grado de cooperación entre los usuarios y sus autoridades, con lo que se logra la equidad en el acceso al recurso; esto con el establecimiento de fechas para el riego, diseñadas según un plan de usos y costumbres, para que los representantes de los ejidos y pequeña propiedad acudan con el secretario o presidente del consejo directivo a entregarle un oficio donde solicitan el riego en fechas acordadas por usuarios, autoridades locales y la asociación civil.

Los usuarios son los responsables de cuidar el agua y no desperdiciarla, porque saben que otros la necesitan, así que entre ellos se van pasando el agua una vez que han regado su parcela. Esto significa que entre tres o seis deben aprovechar en un día el agua que corre por un canal secundario (derivado del principal de riego). La 
distribución se hace por tiempos, es decir, el primer turno le corresponde a quienes tienen su terreno cerca del canal (el riego dura de seis a ocho horas). Los del segundo turno tienen más horas de riego, por la distancia que recorre el agua del canal a sus terrenos. Los usuarios se ponen de acuerdo con los jueces de agua, delegados de riego y comisariados ejidales sobre cuánta agua deben dejar correr por los canales, para que el recurso les beneficie a todos y no se desperdicie.

La cohesión entre usuarios se ejemplifica en el proceso de solicitud de agua ante sus autoridades. Primero, los regantes tienen que estar registrados en el padrón de usuarios; segundo, deben unirse para solicitar el agua al comité que ellos legitiman y tercero, deben contar con su recibo de pago y tener cubiertas sus faenas comunitarias.

La operación del sistema de riego requiere de un grado de coordinación entre las autoridades locales y foráneas respecto al mantenimiento de la infraestructura hidráulica antes de que inicie el riego, el consejo directivo establece la mayor parte del manejo del sistema, aunque normalmente no reglamenta las tareas de mantenimiento, conservación y cuidado de la infraestructura.

Los regantes se encargan de llevar el agua desde los canales concesionados a los terrenos de cultivo, el Estado se mantiene al margen de las estrategias adoptadas por los nuevos regantes en la administración, operación y manejo del sistema. Sin embargo, sí interviene en la designación de las autoridades que lo conforman, como en la elección de los delegados de riego y del consejo directivo. Esta unidad de riego oficialmente está al margen de todo apoyo gubernamental, sin embargo en la práctica, el Estado sigue teniendo injerencia en la administración del sistema no de manera operacional, pero sí en la presencia de sus autoridades y en el papel que juegan como legitimadores de los consejos directivos.

La aproximación teórica al conflicto social que discute la prominencia de efectos contenedores de problemas, como la cohesión y la cooperación, permitió descubrir y describir que en la administración, operación y manejo del Sistema de Riego Tepetitlán se vincula el proceso de interacción entre las 46 comunidades para el aprovechamiento del recurso agua. 
El tipo de agricultura de subsistencia de la zona de riego del sistema Tepetitlán está amenazado por fuerzas externas, que evidentemente tienen que ver con las políticas nacionales en materia de agua y también las agrarias, como el caso de la trasferencia de este distrito de riego a los usuarios.

\section{Conclusiones}

En el Sistema de Riego Tepetitlán, el conflicto es parte inherente a la trasferencia de distritos de riego por parte del Estado, que ocurrió en un ambiente donde los usuarios no fueron consultados sobre las condiciones en que sería entregado no sólo el sistema de riego como tal (desde los canales de riego a las comunidades beneficiadas), sino la red hidráulica, que engloba el saber o aprender a administrarla.

Después de la trasferencia, la administración del Sistema de Riego Tepetitlán presenta singularidades, entre ellas la existencia de dos consejos directivos que lo administran, los problemas entre ellos y la participación del Estado como espectador de los conflictos surgidos a raíz de la política hidráulica de trasferencia.

Las partes involucradas en el conflicto están relacionadas con la administración del sistema, ello de inmediato promueve en los ejidos y pequeñas propiedades la necesidad de acuerdos, pues el agua es un recurso compartido. La gestión del agua brinda la oportunidad a los regantes, autoridades comunitarias y multicomunitarias de enfrentar el conflicto que sostienen las asociaciones civiles, mediante lazos de cooperación y cohesión para evitar el colapso del sistema.

La cooperación en el regadío de Tepetitlán se presenta cuando los implicados en el riego asumen sus derechos, que incluyen tener acceso al agua, y las obligaciones como cumplir con la limpieza, cuidado de la infraestructura hidráulica y apoyo a las autoridades en la distribución del agua, dicha cooperación entre ejidos promueve estabilidad en el sistema de regadío.

El elemento cohesionador también es favorable, al integrarse los regantes como autoridades en la administración buscan consensuar 
cada una de las actividades que realizan, de tal forma que los usuarios consideran ecuánime la actuación de sus autoridades.

El conflicto presente entre asociaciones civiles encuentra un punto de equilibrio en las autoridades comunitarias, las cuales logran contener los enfrentamientos al mostrar su capacidad para organizar a los usuarios en la limpia de canales, pago del riego, cobro de multas y la conservación del sistema.

Las asociaciones civiles que afrontan la administración del sistema de riego han originado una reestructuración organizativa en el riego. La presencia de organizaciones de usuarios en Tepetitlán después de la trasferencia no son adjudicables al Estado, constituyen más bien una de las múltiples respuestas de los regantes a la política hidráulica de trasferir los distritos de riego a los usuarios, que muestra las incompatibilidades de administrar un sistema. El Estado promueve una estructura rígida donde sólo una autoridad superior puede contener los conflictos, y los regantes optan por la flexibilidad, y pugnan por la apertura de las autoridades al diálogo antes que al enfrentamiento.

En este sentido, el Estado debe reforzar las organizaciones autogestivas legitimando las capacidades de las autoridades locales, que en este espacio de regadío dan muestra de su inminente preocupación por tratar de minimizar el conflicto entre regantes y asociaciones civiles.

Recibido en octubre 2010 Aceptado en diciembre de 2010

\section{Bibliografía}

CONAGUA. 2002. Programa Hidráulico Regional: Lerma-SantiagoPacífico, 2000-2006.

Cressier, Patricia. 1995. Hidráulica rural tradicional de origen medieval en Andalucía y Marruecos. Elementos de análisis práctico. En El agua. Mitos, ritos y realidades, coordinado por González Alcantud 
y A. Malpica Cuello, 255-282. España: Anthropos/Diputación Provincial de Granada.

Fernea, A. Robert. 1997. El conflicto en el regadío. En Antología sobre pequeño riego, editado por Tomás Martínez y Jacinta Palerm, 170180. Texcoco: Colegio de Postgraduados.

Gleick, Peter. 1994. La reducción de los riesgos de conflictos por los recursos de agua dulce en el Oriente Medio. En Agua y paz en el Oriente Medio, editado por J. Isaac y H. Shuval, 41-54. Países Bajos: Editores de Elsevier.

1993. El agua y los conflictos: recursos de agua fresca y la seguridad internacional. Seguridad Internacional 18 (1): 79-112.

Giner, J. 2009. Teorías del conflicto social. http://147.96.1.15info eurotheo/diccionario/C/conficto_social_teorias.pdf $(12 \mathrm{de}$ marzo de 2009).

Hevia de la Jara, Felipe. 2009. Relaciones sociedad-Estado: análisis interactivo para una antropología del Estado. Espiral xv: 45. http: //redalyc.uaemex.mx/src/inicio/ArtPdfRed.jsp?iCve $=13811771002$ (12 de enero de 2010).

Hunt, Robert. 1997. Sistemas de riego por canales, tamaño del sistema y estructura de autoridad. En Antología sobre pequeño riego, volumen i, editado por Tomás Martínez y Jacinta Palerm, 185-220. Texcoco: Colegio de Postgraduados.

Kelly, W. 1983. Concepts in the Anthropological Study of Irrigation. American Anthropologist 85: 880-886. http://research.yale.edu/ wwkelly/pubs-archive/wwk_1983_AA_85-4.pdf (12 de enero de 2010).

Maass, Arthur y Raymond L. Anderson. 1997. Y el desierto se regocijará... Conflicto, crecimiento y justicia en las zonas áridas. En 
Antología sobre pequeño riego, volumen I, editado por Tomás Martínez y Jacinta Palerm, 261-284. Texcoco: Colegio de Postgraduados.

Martínez, Tomás y Jacinta Palerm Viqueira (editores). 1997. Antología sobre el pequeño riego. Texcoco: Colegio de Potsgraduados.

Méndez Álvarez, C. 2000. Un marco teórico para el concepto de cultura cooperativa. Bogotá: Facultad de Altos Estudios de Administración y Negocios, Universidad del Rosario.

Millon R., C. Hall y M. Díaz. 1997. El conflicto en el sistema de Teotihuacán moderno. En Antología sobre pequeño riego, volumen I, editado por Tomás Martínez y Jacinta Palerm, 71-121. Texcoco: Colegio de Postgraduados.

Montes de Oca, A., Jacinta Palerm y Cristina Chávez. 2010. Las vicisitudes de la trasferencia del sistema de riego Tepetitlán, Estado de México. Agricultura, Sociedad y Desarrollo viI (3): 109-135.

Murillo, Sergio V., y R. Romero. 2000. Comunicación y apoyo en la trasferencia del módulo Tepetitlán en el distrito de riego 033, Estado de México. Anuario del Instituto Mexicano de Tecnología del Agua (IMTA), 138-143. Morelos: IMTA.

Palerm Viqueira, Jacinta. 2008. Distritos de riego en México, algunos mitos. Boletín del Archivo Histórico del Agua 13 (38): 49-69.

2005a. Políticas del Estado para la administración y gobierno de sistemas de riego y redes hidráulicas. En El agua en la historia de México, editado por Juan Manuel Durán, Martín Sánchez y Antonio Escobar, 263-288. Michoacán: Centro Universitario de Ciencias Sociales y Humanidades de la Universidad de Guadalajara/El Colegio de Michoacán.

2005b. Gobierno y administración de sistema de riego. región y sociedad XVII (34): 3-33. 
2004. Prólogo: Reglamentos de aguas y reglamentación de aguas de propiedad nacional. En Catálogo de reglamentos de agua en México siglo xx, coordinado por ídem., Israel Sandré y Benito Rodríguez: 1-40. México. Archivo Histórico del Agua, Centro de Investigación de Estudios Superiores en Antropología Social, coNAGUA.

2000a. Organización social y agricultura de riego. En Antología sobre pequeño riego, volumen II, editado por ídem., y Tomás Martínez, 55-89. Texcoco: Colegio de Postgraduados.

2000b. Administración de sistemas de riego: tipos de autogestión. Ponencia presentada en el x Congreso nacional de irrigación, Asociación Nacional de Especialistas en Irrigación, Chihuahua.

Tomás Martínez y Francisco Escobedo. 2000. Modelo de investigación: organización social de sistemas de riego en México. En Antología sobre pequeño riego, volumen II. Organizaciones autogestivas, editado por Jacinta Palerm y Tomás Martínez, 31-61. Texcoco: Colegio de Postgraduados

Pérez, Luz N. 1994. ¿Y venimos a entregarles el agua?, burócratas y usuarios en el proceso de trasferencia del distrito de riego 097 Lázaro Cárdenas, región de la tierra caliente michoacana. Ponencia presentada en el coloquio Las disputas por el México rural. Transformaciones de identidades, prácticas y proyectos, Michoacán.

Rendón Pimentel, Luis. 2008. Los distritos de riego. Infraestructura y funcionamiento. Ponencia presentada en el xx Congreso nacional de hidráulica. Asociación Mexicana de Hidráulica, Guadalajara.

Wester, P., S. Vargas y E. Mollard. 2004. Negociación y conflicto por el agua superficial en la cuenca Lerma-Chapala: actores, estrategias, alternativas y perspectivas (1990-2004). Ponencia pre- 
sentada en el tercer Encuentro de investigadores del agua en la cuenca Lerma-Chapala-Santiago, Jalisco.

Vaydianathan, A. 2000. Instituciones de control del agua y agricultura: una perspectiva comparativa. En Aventuras con el agua, editado por Jacinta Palerm y Tomás Martínez, 79-162. Texcoco: Colegio de Postgraduados.

Wittfogel, Karl. 1966. Despotismo oriental, estudio comparativo del poder totalitario. Madrid: Guadarrama.

Wolf, Aaron, Annika Kramer, Alexander Carius y Geoffrey D. Dabelko. 2005. Gestionando conflictos por el agua y cooperación. En La situación del mundo: redefiniendo la seguridad mundial. Informe anual del Worldwatch Institute sobre el progreso hacia una sociedad sostenible, editado por Michael Renner, Hilary French y Erik Assadourian, 155-1 78. Barcelona: Icaria; Centro de Investigación para la Paz. 


\section{Anexo}

\section{Entrevistas}

\begin{tabular}{|l|l|l|}
\hline Nombre & Autoridad & Fecha \\
\hline Bernardino & $\begin{array}{l}\text { Comisario ejidal de Santa Ana La } \\
\text { Ladera y vocal del Consejo de } \\
\text { Vigilancia del Grupo Boxipe }\end{array}$ & 11 de febrero de 2009 \\
\hline Castro & $\begin{array}{l}\text { Presidente de la asociación civil } \\
\text { (2002-2004) “Unión Rural } \\
\text { de la Presa Tepetitlán” }\end{array}$ & 13 de marzo de 2009 \\
\hline Garduño & $\begin{array}{l}\text { Delegado de riego de San Miguel } \\
\text { Enyege }\end{array}$ & 14 de agosto de 2008 \\
\hline Nieves & $\begin{array}{l}\text { Primer vocal del Consejo Directivo } \\
\text { Grupo Mextepec }\end{array}$ & 14 de agosto de 2009 \\
\hline Sabino & Usuario de San Isidro Boxipe & 31 de septiembre de 2008 \\
\hline Sánchez & Delegado de riego de San Isidro Boxipe & 16 de febrero de 2009 \\
\hline Mejía Pedrero & $\begin{array}{l}\text { Subgerente de Ingeniería, gerencia } \\
\text { estatal, en el Estado de México }\end{array}$ & 26 de marzo de 2009 \\
\hline $\begin{array}{l}\text { José Aurelio } \\
\text { Monroy }\end{array}$ & $\begin{array}{l}\text { Subgerente de operación del distrito } \\
\text { de riego 033, Estado de México }\end{array}$ & 24 de marzo de 2009 \\
\hline $\begin{array}{l}\text { Raúl Rivas } \\
\text { Castillo }\end{array}$ & $\begin{array}{l}\text { Jefe de distritos de riego 33, 44, 73, } \\
88 \text { y 96, Estado de México }\end{array}$ & 12 de marzo de 2009 \\
\hline
\end{tabular}


